\title{
A Turning Point for Scholarly Osteopathic Publications
}

Felix J. Rogers, DO

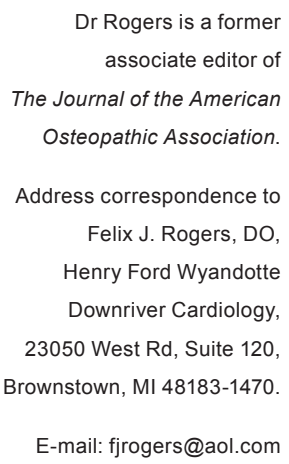

E-mail: fjrogers@aol.com

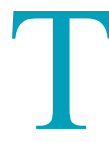

he resignation of Gilbert E. D'Alonzo Jr, $\mathrm{DO}$, as editor in chief of the American Osteopathic Association (AOA) ${ }^{1}$ presents 2 challenges, each of them daunting. Clearly the first challenge is to find his replacement. The AOA has had just a handful of editors in its history, and Dr D'Alonzo will be leaving a remarkable legacy. Appropriately, a search committee has initiated a national search for his successor.

For this first challenge, one easy starting point for the search committee would be to begin with a list of some of the attributes and accomplishments of Dr D'Alonzo. He is a superb clinician, a nationally renowned expert in pulmonary medicine, an educator, a researcher, and an author. As editor in chief, he provided sympathetic and encouraging support for the component of our profession that practices traditional osteopathic manipulative medicine. He called for scientific rigor in all aspects of the AOA publications, from student abstracts to scholarly research articles. He promoted osteopathic medicine to the wider medical community and to the general public through vehicles such as the AOA's custom publications and The DO magazine. And, importantly, he expanded the publication of osteopathic medical research in The Journal of the American Osteopathic Association (JAOA) and extended its scope to include evidence-based clinical reviews, summaries of the worldwide research in manipulative medicine ("The Somatic Connection"), and a section that touches on the humanistic components of the practice of osteopathic medicine ("In Your Words").

The second challenge is to explore and enhance the role of scholarly publications in the overall mission of osteopathic medicine. As a full-fledged medical institution, osteopathic medicine is expected to encompass and embrace clinical service, education, research, and scholarly publications. Traditionally, the success of the osteopathic medical profession has rested on clinical service. The earliest efforts of the AOA were to secure full prac- tice rights for osteopathic physicians; osteopathic medicine's prominent role in health care today rests on our achievements in the delivery of patient care. Recently, the profession has sought to expand its offerings in medical education to help meet the anticipated physician shortage. The number of colleges of osteopathic medicine (COMs) draws on our successful programs for training primary care physicians. Recognizing that our tenets and practices need to be verified by research, the profession has made incremental steps to demonstrate the worth of our educational programs and to document the scientific basis and clinical evidence in support for our practice patterns. The 2013-2022 Strategic Research Plan described by Drs Degenhardt and Standley ${ }^{2}$ represents a comprehensive roadmap to foster research throughout the osteopathic medical profession in language that expresses the urgency of the problem.

Scholarly publications are the obligatory and unifying component for each aspect of clinical practice, education, and research. In one sense, scholarly publication is just like documentation in the patient bedside record, where it is said, "If it's not documented, it didn't happen." Osteopathic medicine has thrived for more than 100 years, but each year it faces increasing demands from patients, third-party payers, and government regulators. Unless we document our effectiveness, confirm our uniqueness, and provide scientific evidence for our distinctive approach to patient care, we will be at risk of losing our place in the medical profession.

The world of medicine has grown increasingly complex, and there is no single, easy fix. Scholarly publications alone cannot meet the needs of the osteopathic medical profession. After all, the role of such scientific reports is to document innovative educational processes, unique and effective clinical treatment, and the scientific basis of osteopathic medical care. If the research isn't there, even the slickest medical publication won't make up for that deficiency. 
However, scholarly publication can be a catalyst for the growth and development of the profession. Our COMs continue to promote the training of primary care physicians and emphasize the role of clinical service by faculty. Generally, COMs do not require scholarly publications or research as part of the tenure process. A shift in focus by the academic centers would lead to a major change in how COMs function and how osteopathic physicians are viewed by the greater scientific community. More importantly, our graduates would be the beneficiaries of such a process, one that enhances a necessary academic orientation to our COMs.

At the same time, we need to recognize that the $J A O A$ has to be considered the first choice for our faculty and clinicians in terms of scholarly publications. There are many options out there, including specialty journals, online publications, and journals with larger circulations or impressive impact factors. That is why this opportunity is a 2-part chal- lenge. It will not be enough just to fill the position of editor in chief with a highly talented individual like Dr D'Alonzo. The next editor also needs to make a similar commitment to Dr D'Alonzo's unceasing efforts to enhance and invigorate the JAOA and the AOA's other publications. To take the JAOA to the next level, we will need a profession-wide commitment to scholarly publications ${ }^{2}$ and the restructuring of our educational and clinical processes - only then can we fulfill our expected role as a major participant in health care today. (doi:10.7556/jaoa .2013.039)

\section{References}

1. Search for a new editor in chief [editorial]. J Am Osteopath Assoc. 2013;113(8):591. doi:10.7556/jaoa.2013.016.

2. Degenhardt BF, Standley PR. 2013-2022 Strategic Plan for Research: a role for everyone in promoting research in the osteopathic medical profession. J Am Osteopath Assoc. 2013;113(9):654-659. doi:10.7556/jaoa.2013.029.

๑) 2013 American Osteopathic Association 